\title{
On defining groups efficiently without using inverses
}

\author{
By C. M. CAMPBELL \\ Mathematical Institute, University of St Andrews, \\ St Andrews KY16 9SS. \\ e-mail: cmc@st-and.ac.uk \\ J. D. MITCHELL \\ Mathematical Institute, University of St Andrews, \\ St Andrews KY16 9SS. \\ e-mail: jamesm@mcs.st-and.ac.uk \\ AND N. RUŠKUC \\ Mathematical Institute, University of St Andrews, \\ St Andrews KY16 9SS. \\ e-mail: nik@mcs.st-and.ac.uk
}

(Received 14 November 2000; revised 11 December 2000)

Abstract

Let $G$ be a group, and let $\langle A \mid R\rangle$ be a finite group presentation for $G$ with $|R| \geqslant|A|$. Then there exists a finite semigroup presentation $\langle B \mid Q\rangle$ for $G$ such that $|Q|-$ $|B|=|R|-|A|$. Moreover, $B$ is either the same generating set or else it contains one additional generator.

\section{Introduction}

The relationships between group and semigroup presentations have been studied for a number of years. For example, Conway [8], in the solution to his problem regarding the Fibonacci group $F(2,5)$, defined by $\langle a, b, c, d, e| a b=c, b c=d, c d=e$, $d e=a, e a=b\rangle$, remarks that the semigroup defined by this presentation is also $F(2,5)$. This led to a more detailed study of Fibonacci groups and semigroups in [6]. Adian [1] initiated the investigation of conditions under which a semigroup $S$ can be embedded into a group $G$ defined by the same presentation; see also [10]. A dual question as to when $S$ contains $G$ as a subsemigroup was considered in [7]. Finally, recent developments in computational group theory based on the use of rewriting systems, as well as the theory of automatic groups, rely on considering semigroup generating sets and presentations for groups; see $[\mathbf{9}, \mathbf{1 8}]$.

Another classical topic in combinatorial group theory is that of minimal and efficient presentations; see [5, 12, 13, 16, 19]. For semigroups, Neumann [15] remarks that it is easy to construct finite semigroups defined by $n$ (independent) generators 
and $n$ relations, and a more comprehensive study of minimality and efficiency was started in [2]. One of the recurring themes of this work is the comparison of group and semigroup presentations for a given group. The purpose of this paper is to prove a general result which, in effect, says that semigroup and group presentations are equally powerful for defining all finite groups (and many infinite ones). In order to state this result precisely we need to introduce some technical concepts and notation.

A (semigroup or group) presentation is a pair $\langle A \mid R\rangle$ of formal generators and relations. Relations are expressions of the form $u=v$ where, in the case of semigroup presentations, $u$ and $v$ are non-empty words over $A$, and in the case of group presentations they are (possibly empty) words over $A \cup A^{-1}$. Every semigroup presentation $\langle A \mid R\rangle$ defines a semigroup; it can be conveniently thought of as the set of all non-empty words over $A$, with two words being equal if and only if one can be obtained from the other by applying relations from $R$. Similarly, a group presentation $\langle A \mid R\rangle$ defines the group consisting of all words over $A \cup A^{-1}$ with two words being equal if and only if one can be obtained from the other by applying relations from $R$ and $a a^{-1}=a^{-1} a=1(a \in A)$. Intuitively, a semigroup presentation for a group defines that group without using inverses and any consequences of their existence (such as cancellation). For basic facts about semigroup and group presentations see any standard introductory texts on semigroups and groups, such as $[\mathbf{1 1}, \mathbf{1 7}]$.

Main Theorem. If $\langle A \mid R\rangle$ is a finite presentation for a group $G$ such that $|R| \geqslant|A|$, then there exists a semigroup presentation $\langle B \mid Q\rangle$ which also defines $G$ and satisfies $|Q|-|B|=|R|-|A|$.

The number $|R|-|A|$ for a finite (semigroup or group) presentation $\mathscr{P}=\langle A \mid R\rangle$ is called the deficiency of $\mathscr{P}$ and is denoted by $\operatorname{def}(\mathscr{P})$. (This definition follows [2] and [13]; some more classical sources such as [14] use $|A|-|R|$.) The semigroup deficiency $\operatorname{def}_{\mathrm{S}}(S)$ of a finitely presented semigroup $S$ and the group deficiency $\operatorname{def}_{\mathrm{G}}(G)$ of a finitely presented group $G$ are defined as

$$
\begin{aligned}
\operatorname{def}_{\mathrm{S}}(S) & =\min \{\operatorname{def}(\mathscr{P}) \mid \mathscr{P} \text { is a finite semigroup presentation for } S\} \\
\operatorname{def}_{G}(G) & =\min \{\operatorname{def}(\mathscr{P}) \mid \mathscr{P} \text { is a finite group presentation for } G\} .
\end{aligned}
$$

Universal considerations show that every semigroup presentation for a group $G$ is also a group presentation for $G$. Therefore, for every finitely presented group we have $\operatorname{def}_{G}(G) \leqslant \operatorname{def}_{S}(G)$. It is easy to see that strict inequality holds for every group $G$ with $\operatorname{def}_{G}(G)<0$. Indeed, if $\langle A \mid R\rangle$ is a semigroup presentation for a group $G$ then $R$ must contain relations of the form $a=v$ for all $a \in A$, and hence $\operatorname{def}_{S}(G) \geqslant 0$. With this in mind, our Main Theorem can be restated as follows:

Main Theorem. Let $G$ be a finitely presented group. If $\operatorname{def}_{G}(G) \geqslant 0$ (in particular if $G$ is finite) then $\operatorname{def}_{S}(G)=\operatorname{def}_{G}(G)$. Otherwise if $\operatorname{def}_{G}(G)<0$ then $\operatorname{def}_{S}(G)>\operatorname{def}_{G}(G)$.

In fact, we prove more: given a group presentation of non-negative deficiency defining a group $G$, we show how to construct a semigroup presentation for $G$ of the same deficiency, either on the same generating set (Proposition 2.4) or on the same generating set plus one additional generator (Proposition 2.5). 


\section{Proof of the Main Theorem}

We prove the main theorem in two steps. First, we find a family of semigroup presentations which define groups, and then show how to transform a group presentation of non-negative deficiency defining a given group $G$ into a semigroup presentation from this family which has the same deficiency and also defines $G$. The first step is accomplished in the following:

Proposition $2 \cdot 1$. The semigroup presentation

$$
\mathscr{P}=\left\langle a_{1}, a_{2}, \ldots, a_{n} \mid a_{1}=a_{2} \beta_{1} a_{2} a_{1}, a_{2}=a_{3} \beta_{2} a_{3}, a_{3}=a_{4} \beta_{3} a_{4}, \ldots, a_{n}=a_{1} \beta_{n} a_{1}, R\right\rangle,
$$

where $n \geqslant 1, \beta_{1}, \beta_{2}, \ldots, \beta_{n}$ are arbitrary words over $\left\{a_{1}, a_{2}, \ldots, a_{n}\right\}$ and $R$ is an arbitrary set of relations, defines a group.

Remark 2.2. For $n=1$ the above presentation is taken to be of the form $\langle a| a^{k}=$ $a, R\rangle(k \geqslant 2)$, i.e. it is a semigroup presentation for a finite cyclic group.

In the proof it will be convenient to make use of Green's relations $\mathscr{R}$ and $\mathscr{L}$ on a semigroup $S$. Recall that for $s, t \in S$ we write $s \mathscr{R} t$ if $s \in t S \cup\{t\}$ and $t \in s S \cup\{s\}$. The relation $\mathscr{L}$ is defined dually. It is well known that semigroup is a group if and only if it has a unique $\mathscr{R}$-class and a unique $\mathscr{L}$-class. For further details see [11].

Lemia 2.3. Let $S$ be a non-monogenic semigroup and let $A$ be a generating set for $S$.

(i) If $a b \mathscr{R} a$ (respectively $a b \mathscr{L} b$ ) for all $a, b \in A$ then aw $\mathscr{R} a$ (respectively $w b \mathscr{L} b$ ) for all $a \in A$ and $w \in S$.

(ii) If for any $a, b \in A$ there exists $s \in S$ such that $a s=b$ (respectively $s a=b)$ then $S$ has only one $\mathscr{R}$-class (respectively $\mathscr{L}$-class).

Proof. (i) The assertion follows by a straightforward induction on the length of $w$ as a product of generators from $A$; see also [6, lemma 2.5].

(ii) By assumption there exist $u \in S$ such that $a=a u$. If $u=c_{1} c_{2} \cdots c_{k}(k \leqslant 1$, $\left.c_{i} \in A\right)$ then write $c_{1}=b v$ so that $a=a u=a b v c_{2} \cdots c_{k}$. Therefore $a \in a b S$ and since obviously $a b \in a S$ it follows that $a \mathscr{R} a b$. The assertion now follows from (i).

Proof of Proposition 2.1. Let $S$ denote the semigroup defined by the presentation $\mathscr{P}$ and let $A=\left\{a_{1}, a_{2}, \ldots, a_{n}\right\}$. We prove the proposition by showing that $S$ has a single $\mathscr{R}$-class and a single $\mathscr{L}$-class. From the first $n$ relations in $\mathscr{P}$ we see that for every $i=1, \ldots, n$ there exists a word $u_{i}$ such that $a_{i-1}=a_{i} u_{i}$ (we reduce subscripts modulo $n$, if necessary, so that for $i=1$, we have $a_{n}=a_{1} u_{1}$ ). But then, for $a_{i}, a_{j} \in A$ we have $a_{i}=a_{j} u_{j} u_{j-1} \cdots u_{i+1}$, and it follows from Lemma $2 \cdot 3$ (ii) that $S$ has only one $\mathscr{R}$-class. In other words, $S$ is right simple. Note that

$$
\left(a_{2} \beta_{1} a_{2}\right) a_{j}=a_{2} \beta_{1} a_{2} a_{1} u_{1} u_{n} \cdots u_{j+1}=a_{1} u_{1} u_{n} \cdots u_{j+1}=a_{1}
$$

for $j=1, \ldots, n$. But then

$$
a_{j}=a_{2} \beta_{1} a_{2} a_{j}=a_{2} \beta_{1} a_{3} \beta_{2} a_{3} a_{j}=\cdots=\left(a_{2} \beta_{1} a_{3} \beta_{2} \cdots \beta_{i-2} a_{i} \beta_{i-1}\right) a_{i} a_{j},
$$

and so $a_{i} a_{j} \mathscr{L} a_{j}$ for any $a_{i}, a_{j} \in A$. We have not yet shown that $a_{i} \mathscr{L} a_{j}$ but from Lemma $2 \cdot 3(\mathrm{i})$ it follows that there are only finitely many $\mathscr{L}$-classes in $S$. We see that $S$ is a completely simple right simple semigroup. From the proof of the ReesSuschkewitsch theorem, we have that $S \cong G \times \mathscr{Z}$, where $G$ is a group and $\mathscr{Z}$ is a right zero semigroup, see [11, theorems $3 \cdot 2 \cdot 3,3 \cdot 3 \cdot 1$ and exercises $2 \cdot 6 \cdot 6,3 \cdot 8 \cdot 11]$. 
Now $\mathscr{Z}$ is a natural homomorphic image of $S$, and is defined by

$$
\begin{aligned}
\left\langle a_{1}, a_{2}, \ldots, a_{n}\right| a_{1} & =a_{2} \beta_{1} a_{2} a_{1}, a_{2}=a_{3} \beta_{2} a_{3}, \\
a_{3} & \left.=a_{4} \beta_{3} a_{4}, \ldots, a_{n}=a_{1} \beta_{n} a_{1}, R, a_{i} a_{j}=a_{j} \quad(1 \leqslant i, j \leqslant n)\right\rangle .
\end{aligned}
$$

But this is clearly equivalent to

$$
\left\langle a_{1}, a_{2}, \ldots, a_{n} \mid a_{1}^{2}=a_{1}=a_{2}=\ldots=a_{n}\right\rangle ;
$$

in other words $\mathscr{Z}$ is trivial. This implies that $S$ is a group, as required.

Our next result shows how to transform a group presentation, satisfying certain conditions, into a semigroup presentation defining the same group, over the same generating set and with the same deficiency.

Proposition 2.4. Let $G$ be the group defined by the finite group presentation $\mathscr{P}=$ $\langle A \mid R\rangle$ where $|R| \geqslant|A|$ and let $A$ be a semigroup generating set for $G$. In addition, assume that $R$ contains a relation of the form $E=1$, where $E$ is a word which contains no inverses of generators, but which contains every generator at least once, and also contains the square of at least one generator. Then $G$ has a semigroup presentation $\langle A \mid Q\rangle$ with $|Q|=|R|$.

Proof. Let $A=\left\{a_{1}, a_{2}, \ldots, a_{n}\right\}$ and without loss of generality let $R=\left\{E=1, u_{2}=\right.$ $\left.1, \ldots, u_{n}=1, R^{\prime}\right\}$. Also without loss of generality assume that $E$ contains a subword $a_{2}^{2}$. For $i=1, \ldots, n$ let $E_{i}^{(l)}$ (respectively $E_{i}^{(r)}$ ) denote the cyclic permutation of $E$ beginning (respectively ending) with $a_{i}$. Let $E_{2}^{(t)}$ denote the permutation of $E$ beginning and ending with $a_{2}$. We replace the relation $E=1$ by the relation $E_{2}^{(t)} a_{1}=a_{1}$ and each of the relations $u_{i}=1$ by $E_{i+1}^{(l)} u_{i} a_{i} E_{i+1}^{(r)}=a_{i}$. This gives the presentation

$$
\begin{gathered}
\mathscr{P}^{\prime}=\left\langle a_{1}, a_{2}, \ldots, a_{n}\right| E_{2}^{(t)} a_{1}=a_{1}, E_{3}^{(l)} u_{2} a_{2} E_{3}^{(r)}=a_{2}, E_{4}^{(l)} u_{3} a_{3} E_{4}^{(r)}=a_{3}, \ldots, \\
\left.E_{n}^{(l)} u_{n-1} a_{n-1} E_{n}^{(r)}=a_{n-1}, E_{1}^{(l)} u_{n} a_{n} E_{1}^{(r)}=a_{n}, R^{\prime \prime}\right\rangle,
\end{gathered}
$$

where $R^{\prime \prime}$ is obtained from $R^{\prime}$ by replacing every occurrence of an inverse by a word that contains no inverses and every occurrence of the empty words by $E$. More precisely, we replace $a_{i}^{-1}$ by $E_{i}^{(r)} a_{i}^{-1}$, which contains no inverses since the last two letters cancel. Obviously, $\mathscr{P}^{\prime}$ as a group presentation still defines $G$. On the other hand, $\mathscr{P}^{\prime}$ contains no inverses, i.e. it is a semigroup presentation. Moreover, it has the form given in Proposition $2 \cdot 1$, and hence the semigroup defined by it is a group, which is then necessarily isomorphic to $G$.

If our starting group presentation does not contain a relation $E=1$, where $E$ is as above, we can still transform it into one that does, by adding one generator and one relation.

Proposition 2.5. Let $\mathscr{P}=\left\langle a_{1}, a_{2}, \ldots, a_{n} \mid R\right\rangle$ be a group presentation of nonnegative deficiency which defines a group $G$. Then $G$ has a semigroup presentation $\mathscr{Q}=\left\langle a_{1}, a_{2}, \ldots, a_{n}, b \mid Q\right\rangle$ such that $\operatorname{def}(\mathscr{Q})=\operatorname{def}(\mathscr{P})$ and $b$ represents the product $a_{n}^{-2} a_{n-1}^{-1} \cdots a_{1}^{-1}$.

Proof. We obtain a new group presentation by adding the generator $b$ and the relation $a_{1} a_{2} \cdots a_{n-1} a_{n}^{2} b=1$ to the presentation $\mathscr{P}$. Note that the set $\left\{a_{1}, a_{2}, \ldots, a_{n}, b\right\}$ 
is a semigroup generating set for $G$. This new presentation clearly defines $G$ and satisfies the conditions of Proposition $2 \cdot 4$. Hence we may find the semigroup presentation 2 using Proposition $2 \cdot 4$, as required.

Note that every finite group $G$ has non-negative deficiency, and that every group generating set for $G$ is also a semigroup generating set. However, it is not known whether, given a group presentation $\langle A \mid R\rangle$ for $G$, one can always find a semigroup presentation for $G$ on the same generating set and of the same deficiency. In particular, it is not known whether there is a group presentation for $G$ on the generators $A$, having the same deficiency as $\langle A \mid R\rangle$ and containing a relation $E=1$ of the form described in Proposition $2 \cdot 4$.

\section{Examples}

In $[2,3,4]$, efficient semigroup presentations for various groups are found. The groups considered include the finite abelian groups, dihedral groups, (projective) special linear groups and wreath products. In fact, our Main Theorem implies that a finite group is efficient as a group if and only if it is efficient as a semigroup. Here we illustrate constructions described in Propositions 2.4 and 2.5 by giving two new examples: the wreath products $C_{n} \prec C_{m}$ ( $n$ odd, $m$ even) of two cyclic groups and the projective special unitary group $P S U(3,3)$

Example $3 \cdot 1$. An efficient group presentation for $C_{n} \prec C_{m}$ with $n$ odd and $m$ even is

$\left\langle a_{1}, a_{2} \mid a_{1}^{n} a_{2}^{m}=1,\left(a_{1} a_{2}^{m / 2}\right)^{2}=a_{2}^{m / 2} a_{1} a_{2}^{-m / 2} a_{1},\left[a_{1}, a_{2}^{-i} a_{1} a_{2}^{i}\right]=1(1 \leqslant i \leqslant(m-2) / 2)\right\rangle ;$

see [3] for details. Observe that this presentation satisfies the conditions in Propostion $2 \cdot 4$; more precisely the relation $a_{1}^{n} a_{2}^{m}=1$ contains no inverses, contains both generators and contains the square of a generator. We define $E \equiv a_{1}^{n} a_{2}^{m}$ and take $E_{1}^{(l)} \equiv E_{2}^{(r)} \equiv E, E_{2}^{(t)} \equiv E_{2}^{(l)} \equiv a_{2} a_{1}^{n} a_{2}^{m-1}$ and $E_{1}^{(r)} \equiv a_{1}^{n-1} a_{2}^{m} a_{1}$. Using the prescription in Proposition $2 \cdot 4$ we find the semigroup presentation

$$
\begin{array}{r}
\left\langle a_{1}, a_{2}\right| a_{2} a_{1}^{n} a_{2}^{m-1} a_{1}=a_{1}, a_{1}^{n} a_{2}^{m} a_{1}^{n-1} a_{2}^{m / 2}\left(a_{2}^{m} a_{1}^{n-1}\right)^{2}\left(a_{1} a_{2}^{m / 2}\right)^{3} a_{2} a_{1}^{n-1} a_{2}^{m} a_{1}=a_{2}, \\
\left.a_{1}^{n-1} a_{2}^{m} a_{1}^{n} a_{2}^{m-i} a_{1}^{n-1} a_{2}^{m+i} a_{1}^{n+i} a_{2}^{m-i} a_{1} a_{2}^{i}=a_{1}^{n} a_{2}^{m}(1 \leqslant i \leqslant(m-2) / 2)\right\rangle
\end{array}
$$

that defines $C_{n}<C_{m}$.

Example 3.2. An efficient group presentation for $P S U(3,3)$ is

$$
\left\langle a_{1}, a_{2} \mid a_{1}^{3} a_{2} a_{1}^{3}=a_{2}^{2},\left(a_{2} a_{1}\right)^{2} a_{2}^{2} a_{1}^{-1} a_{2}^{-3} a_{1} a_{2}^{-1} a_{1}^{-3}=1\right\rangle ;
$$

see [12]. Since we have no relation of the type given in Proposition $2 \cdot 4$ we have to add a new generator $b$ representing $a_{2}^{-2} a_{1}^{-1}$. Also we add the relation $a_{1} a_{2}^{2} b=1$ which satisfies Proposition 2.4; we now proceed as in Example $3 \cdot 1$. This gives the semigroup presentation

$$
\begin{aligned}
\left\langle a_{1}, a_{2}, b\right| a_{2} b a_{1} a_{2} a_{1}= & a_{1}, b a_{1} a_{2}^{2} a_{1}^{3} a_{2} a_{1}^{3} b a_{1} a_{2} a_{1} a_{2}^{2} b=a_{2}, \\
& \left.a_{1} a_{2}^{2} b\left(a_{2} a_{1}\right)^{2} a_{2}^{4} b\left(a_{2} b a_{1}\right)^{3} a_{1} a_{2} b a_{1}\left(a_{2}^{2} b\right)^{3} b a_{2}^{2} b a_{1}=b\right\rangle
\end{aligned}
$$

for $P S U(3,3)$. 


\section{REFERENCES}

[1] S. I. Adian. Defining relations and algorithmic problems for groups and semigroups. Proc. Steklov Inst. Math. 85 (1966); translated from Trudy Mat. Inst. Steklov. 85 (1966).

[2] H. Ayik, C. M. Campbell, J. J. O’Connor and N. RušKuc. Minimal presentations and efficiency of groups and monoids. Semigroup Forum 60 (2000), 231-242.

[3] H. Ayik, C. M. Campbell, J. J. O'Connor and N. RušKuc. On the efficiency of wreath product of groups. In: Proceedings of Groups - Korea 98, Y. G. Baik, D. L. Johnson and A. C. Kim (eds.) (Walter de Gruyter, 2000) pp. 39-51.

[4] H. Ayik, C. M. Campbelt, J. J. O'Connor and N. Ruškuc. The semigroup efficiency of groups and monoids. Proc. Roy. Irish Acad. 100A (2000), 171-176.

[5] C. M. Campbell and E. F. Robertson. A deficiency zero presentation for $S L(2, p)$. Bull. London Math. Soc. 12 (1980), 17-20.

[6] C. M. Campbell, E. F. Robertson, N. Ruškuc and R. M. Thomas. Fibonacci semigroups. J. Pure Appl. Algebra 94 (1994), 49-57.

[7] C. M. Campbell, E. F. Robertson, N. Ruškuc and R. M. Thomas. On subsemigroups of finitely presented groups. J. Algebra 180 (1996), 1-21.

[8] J. H. Conway. Solution to Advanced Problem 5327. Amer. Math. Monthly 74 (1967), 91-93.

[9] D. B. A. Epstein, J. W. Cannon, D. F. Holt, S. V. F. Levy, M. S. Paterson and W. P. Thurston. Word Processing in Groups (Jones and Bartlett, 1992).

[10] J. Harlander. Embedding into efficient groups. Proc. Edinb. Math. Soc. 40 (1997), 317-342.

[11] J. M. HowiE. Fundamentals of semigroup theory (Clarendon Press, 1995).

[12] P. E. Kenne. Efficient presentations for three simple groups. Comm. Algebra 14 (1986), $797-$ 800.

[13] L. G. Kovacs. Finite groups with trivial multiplicator and large deficiency. In: Groups - Korea 94, A. C. Kim and D. L. Johnson (eds.) (Walter de Gruyter, 1995), pp. 211-226.

[14] R. C. Lyndon and P. E. Schupp. Combinatorial group theory (Springer-Verlag, 1977).

[15] B. H. Neumann. Some remarks on semigroup presentations. Canad. J. Math. 19 (1967), 1018 1026 .

[16] E. F. Robertson, R. M. Thomas and C. I. Wotherspoon. A class of inefficient groups with symmetric presentation. In: Groups - Korea 94, A. C. Kim and D. L. Johnson (eds.) (Walter de Gruyter, 1995), pp. 277-284.

[17] J. J. Rotman. The Theory of Groups: an Introduction (Allyn and Bacon, 1965).

[18] C. C. Sims. Computation with finitely presented groups (Cambridge University Press, 1994).

[19] J. Weigold. The Schur Multiplier: an elementary approach. In: Groups - St Andrews 1981, C. M. Campbell and E. F. Robertson (eds.) London Mathematical Society Lecture Notes (Cambridge University Press) 71, 1982, 39-51. 